\title{
A formula for the error of finite sinc interpolation with an even number of nodes ${ }^{1}$
}

\author{
Jean-Paul Berrut \\ Department of Mathematics \\ University of Fribourg \\ CH-1700 Fribourg/Pérolles \\ Switzerland \\ jean-paul.berrut@unifr.ch
}

\begin{abstract}
Sinc interpolation is a very efficient infinitely differentiable approximation scheme from equidistant data on the infinite line. We give a formula for the error committed when the function neither decreases rapidly nor is periodic, so that the sinc series must be truncated for practical purposes. To do so, we first complete a previous result for an odd number of points, before deriving a formula for the more involved case of an even number of points.
\end{abstract}

Subject classification: AMS(MOS) 65D05.

Key words: sinc interpolation, infinitely differentiable approximation, equidistant interpolation, error formula.

\section{Introduction}

Approximating a function $f$ from a sample at equidistant abscissae is a classical problem in engineering that gives rise to interesting mathematics and recurrently leads to the publication of survey papers and books, such as [Hig1, But-Spl-Ste, But-Ste, Hig2, Par]. One approach to Shannon's sampling theory takes advantage of the Lagrange property

$$
\operatorname{sinc}(k \pi)= \begin{cases}1, & k=0, \\ 0, & k \in \mathbb{Z} \backslash\{0\},\end{cases}
$$

of the sinc function

$$
\operatorname{sinc}(x):=\frac{\sin x}{x}
$$

\footnotetext{
${ }^{1}$ Work partly supported by the Swiss National Science Foundation under grant $\mathrm{Nr}$ PIOI2 -117244/2.
} 
at the integer multiples of $\pi$ to construct the cardinal interpolant

$$
C(f, h)(x):=\sum_{n=-\infty}^{\infty} \operatorname{sinc}\left[\frac{\pi}{h}\left(x-x_{n}\right)\right] f_{n}
$$

from the sample values $f_{n}:=f\left(x_{n}\right)$ at the bi-infinite sequence of equidistant arguments $x_{n}=n h$ (therefore including zero). If $f$ decays rapidly enough at infinity, Shannon's sampling theorem asserts that $C(f, h)=f$ for $h$ sufficiently small if $f$ is the restriction to $\mathbb{R}$ of a function of exponential type (Paley-Wiener class, [Lun-Bow, p. 22 ff.]), while one has exponential convergence of $C(f, h)$ when $f$ is analytic in a horizontal strip about $\mathbb{R}$ ([LunBow, p. 35] or [Ste, p. 136]). These facts make $C(f, h)$ unarguably the most important infinitely differentiable interpolant between equidistant points on the infinite line and on the circle, where it is the trigonometric interpolant [Ber1].

When applying (1.1) in practice, one must restrict oneself to finite sums, which we shall take here to be symmetric about 0 and make longer and longer to improve accuracy. We shall consider a fixed interval $[-X, X], X \in \mathbb{R}^{+}$, and at first choose as in [Ber3] some $h$ such that $X=N h$ for $N \in \mathbb{N}$ to approximate $C(f, h)$ of (1.1) with the finite interpolant

$$
C_{N}(f, h)(x)=\sum_{n=-N}^{N} \prime \prime f\left(x_{n}\right) \operatorname{sinc}\left[\frac{\pi}{h}\left(x-x_{n}\right)\right], \quad x_{n}=n h, \quad h=X / N,
$$

where the double prime denotes that the first and last terms are halved. This permits to write $C_{N}$ as a function of the difference of two classical quadrature formulae, see $\S 3$. We are interested in the error $C_{N}(f, h)-f$ as a function of $h$ for $f \in C^{q}[-X, X]$ for some $q \in \mathbb{N}$. After completing the error term for a formula given in [Ber3], we derive in $\S 4$ the corresponding one for an even number of $x_{n}$.

\section{Preliminaries on numerical quadrature}

As in [Ber3], our analysis rests on the study of errors in numerical quadrature. We shall use the notation $I^{[a, b]}:=\int_{a}^{b} f(y) d y$ for the usual definite integral and $I_{x}^{[a, b]}:=\operatorname{PV} \int_{a}^{b} \frac{f(y)}{x-y} d y$ for the Cauchy principal value integral. A classical formula for approximating the integral $I^{[0, X]}$ from an equispaced sample $f_{n}:=f\left(x_{n}\right)$ of $f$ at $x_{n}:=n h, h:=\frac{X}{N}$, is the trapezoidal rule $T_{h}\left(I^{[0, X]}\right):=h \sum_{n=0}^{N}{ }^{\prime \prime} f_{n}$; if $f \in C^{2 m+1}[0, X]$ and $f^{(2 m+2)}$ is absolutely integrable on $[0, X]$, $m \in \mathbb{N} \cup\{0\}$, then its error is given by the Euler-Maclaurin formula 


$$
\begin{aligned}
T_{h}\left(I^{[0, X]}\right)-I^{[0, X]} & =\sum_{k=1}^{m+1} a_{2 k} h^{2 k}-\frac{h^{2 m+2}}{(2 m+2) !} \int_{0}^{X} f^{(2 m+2)}(x) \bar{P}_{2 m+2}\left(\frac{x}{h}\right) d x, \\
a_{2 k} & :=\frac{B_{2 k}}{(2 k) !}\left[f^{(2 k-1)}(X)-f^{(2 k-1)}(0)\right]
\end{aligned}
$$

where $\bar{P}_{\ell}$ denotes the 1 -periodic continuation of the Bernoulli polynomial of degree $\ell$ [Ell, Ber2] and the constants $B_{\ell}$ are the Bernoulli numbers [Sch, Ber2]. If $f \in C^{2 m+2}$ the two terms in $h^{2 m+2}$ may be combined to yield $\sum_{k=1}^{m} a_{2 k} h^{2 k}+\mathcal{O}\left(h^{2 m+2}\right)$ [Kin-Che].

If the abscissae do not include the endpoints, as with $x_{n+\frac{1}{2}}:=\left(n+\frac{1}{2}\right) h, n=0, \ldots, N-1$, then a possibility is the midpoint rule $M_{h}\left(I^{[0, X]}\right):=h \cdot \sum_{n=0}^{N-1} f_{n+\frac{1}{2}}$, whose error is

$$
M_{h}\left(I^{[0, X]}\right)-I^{[0, X]}=-\sum_{k=1}^{m+1}\left(1-2^{1-2 k}\right) a_{2 k} h^{2 k}-\frac{h^{2 m+2}}{(2 m+2) !} \int_{0}^{X} f^{(2 m+2)}(x) \bar{P}_{2 m+2}\left(\frac{1}{2}-\frac{x}{h}\right) d x
$$

with the same $a_{2 k}$ as in (2.1) [Dav-Rab, p. 139].

Euler-Maclaurin formulae have been given for Cauchy principal value integrals $I_{x}^{[0, X]}$ as well. Restricting himself to analytic $f$ 's, Hunter [Hun] subtracted an analytically integrable $X$-periodic function with the behavior of $\frac{1}{x-y}$ at $x$ and so that the endpoint values of each of its derivatives coincide to obtain an asymptotic series for $T_{h}\left(I_{x}^{[0, X]}\right)-I_{x}^{[0, X]}$. Lyness [Lyn] later noticed that the subtraction function could simply be taken as $\frac{\pi}{X} f(x) \cot \left[\frac{\pi}{X}(x-y)\right]$ and gave the $\mathcal{O}\left(h^{q}\right)$ term for $f \in C^{q}, q \in \mathbb{N}$ - see (4.6) below. Elliott [Ell] fixed a slight error in Lyness' assumptions. The formulae for $T_{h}$ and $M_{h}$ are derived under the hypothesis that $f \in C^{2 m+1}$ and that $f^{(2 m+2)}$ is absolutely integrable. For $T_{h}$ the formula is

$$
\begin{aligned}
T_{h}\left(I_{x}^{[0, X]}\right)=h \sum_{n=0}^{N \prime} \frac{f_{n}}{x-x_{n}} & =I_{x}^{[0, X]}+\pi f(x) \cot \frac{\pi}{h} x+\sum_{k=1}^{m+1} a_{2 k}(x) h^{2 k} \\
& -\frac{h^{2 m+2}}{(2 m+2) !} \int_{0}^{X} F^{(2 m+2)}(y) \bar{P}_{2 m+2}\left(\frac{y}{h}\right) d y \\
a_{2 k}(x) & :=\frac{B_{2 k}}{(2 k) !}\left[\left(\frac{f(y)}{x-y}\right)^{(2 k-1)}(X)-\left(\frac{f(y)}{x-y}\right)^{(2 k-1)}(0)\right], \\
F(y) & :=\frac{f(y)}{x-y}-\frac{\pi}{X} f(x) \cot \left[\frac{\pi}{X}(x-y)\right] .
\end{aligned}
$$


Hunter also gave the corresponding formula for the midpoint rule, which with the integral term by Lyness reads

$$
\begin{array}{r}
M_{h}\left(I_{x}^{[0, X]}\right)=h \sum_{n=0}^{N-1} \frac{f_{n+\frac{1}{2}}}{x-x_{n+\frac{1}{2}}}=I_{x}^{[0, X]}-\pi f(x) \tan \frac{\pi}{h} x-\sum_{k=1}^{m+1}\left(1-2^{1-2 k}\right) a_{2 k}(x) h^{2 k} \\
-\frac{h^{2 m+2}}{(2 m+2) !} \int_{0}^{X} F^{(2 m+2)}(y) \bar{P}_{2 m+2}\left(\frac{1}{2}-\frac{y}{h}\right) d y
\end{array}
$$

with the $a_{2 k}(x)$ and $F(y)$ of (2.3). (Our signs do not match those of [Hun], [Lyn] or [Ell] for we integrate $\frac{f(y)}{x-y}$ instead of $\frac{f(y)}{y-x}$, which changes all signs, including that of the residue in [Hun].)

We shall be concerned with formulae for symmetric integrals $I_{x}^{[-X, X]}=\operatorname{PV} \int_{-X}^{X} \frac{f(y)}{x-y} d y$. To obtain them, we shall modify the interval to $[0,2 X]$ by changing the variable to $t:=y+X$ and defining for every function $s(t)$ its shifted $\widehat{s}(t):=s(t-X)$, so that $I_{x}^{[-X, X]}$ becomes $\mathrm{PV} \int_{0}^{2 X} \frac{\widehat{f}(t)}{x+X-t} d t$. Then by $(2.3)$

$$
\begin{aligned}
& T_{h}\left(I_{x}^{[-X, X]}\right)-I_{x}^{[-X, X]}= \pi \widehat{f}(x+X) \cot \left[\frac{\pi}{h}(x+X)\right]+\sum_{k=1}^{m+1} \widehat{a}_{2 k}(x+X) h^{2 k} \\
&-\frac{h^{2 m+2}}{(2 m+2) !} \int_{0}^{2 X} \widehat{F}_{2}^{(2 m+2)}(t) \bar{P}_{2 m+2}\left(\frac{t}{h}\right) d t, \\
& \widehat{a}_{2 k}(x):=\frac{B_{2 k}}{(2 k) !}\left[\left(\frac{\widehat{f}(t)}{x-t}\right)^{(2 k-1)}(2 X)-\left(\frac{\hat{f}(t)}{x-t}\right)^{(2 k-1)}(0)\right], \\
& \widehat{F}_{2}(t):=\frac{\widehat{f}(t)}{x+X-t}-\frac{\pi}{2 X} \widehat{f}(x+X) \cot \left[\frac{\pi}{2 X}(x+X-t)\right] .
\end{aligned}
$$

But $\frac{2 X}{h}=N$, thus $\frac{X}{h}=\frac{N}{2}$, so that

$$
\begin{aligned}
T_{h}\left(I_{x}^{[-X, X]}\right)- & I_{x}^{[-X, X]}=\pi f(x) \cot \left[\frac{\pi}{h} x+\frac{N}{2} \pi\right]+\sum_{k=1}^{m+1} \widehat{a}_{2 k}(x+X) h^{2 k} \\
& -\frac{h^{2 m+2}}{(2 m+2) !} \int_{-X}^{X} \widehat{F}_{2}^{(2 m+2)}(y+X) \bar{P}_{2 m+2}\left(\frac{y}{h}+\frac{N}{2}\right) d y, \\
\widehat{a}_{2 k}(x) & :=\frac{B_{2 k}}{(2 k) !}\left[\left(\frac{f(t-X)}{x-X-(t-X)}\right)^{(2 k-1)}(2 X)-\left(\frac{f(t-X)}{x-X-(t-X)}\right)^{(2 k-1)}(0)\right], \\
\widehat{F}_{2}(y+X) & :=\frac{f(y)}{x+X-y-X}-\frac{\pi}{2 X} \widehat{f}(x+X) \cot \left[\frac{\pi}{2 X}(x-y)\right] .
\end{aligned}
$$


If $N$ is even, the $\pi$-periodicity of the cotangent and the 1-periodicity of $\bar{P}_{2 m+2}$ have formula (2.3) still hold with $-X$ in place of 0 and double interval length:

$$
\begin{aligned}
T_{h}\left(I_{x}^{[-X, X]}\right)-I_{x}^{[-X, X]} & =\pi f(x) \cot \frac{\pi}{h} x+\sum_{k=1}^{m+1} a_{2 k}(x) h^{2 k} \\
& -\frac{h^{2 m+2}}{(2 m+2) !} \int_{-X}^{X} F_{2}^{(2 m+2)}(y) \bar{P}_{2 m+2}\left(\frac{y}{h}\right) d y, \\
a_{2 k}(x) & :=\frac{B_{2 k}}{(2 k) !}\left[\left(\frac{f(y)}{x-y}\right)^{(2 k-1)}(X)-\left(\frac{f(y)}{x-y}\right)^{(2 k-1)}(-X)\right], \\
F_{2}(y) & :=\frac{f(y)}{x-y}-\frac{\pi}{2 X} f(x) \cot \left[\frac{\pi}{2 X}(x-y)\right] .
\end{aligned}
$$

This remains true for the error of the midpoint rule.

When $N$ is odd, the derivative-free term becomes $\pi f(x) \cot \left[\frac{\pi}{h} x+\frac{\pi}{2}\right]=-\pi f(x) \tan \frac{\pi}{h} x$; moreover, $\bar{P}_{2 m+2}\left(\frac{y}{h}+\frac{N}{2}\right)=\bar{P}_{2 m+2}\left(\frac{1}{2}-\frac{y}{h}\right)$, since the Bernoulli polynomials with even degree are even with respect to $1 / 2$. (We call a function $s$ even with respect to $a$ when $s(a-x)=$ $s(a+x)$.) Those are the corresponding expressions in the error of the midpoint rule. The same calculation leading to (2.5), but for the midpoint rule, results in the derivative-free $\operatorname{term}-\pi f(x) \tan \left[\frac{\pi}{h}(x+X)\right]=\pi f(x) \cot \left(\frac{\pi}{h} x\right)$ and $\bar{P}_{2 m+2}\left(\frac{y}{h}\right)$ of the trapezoidal error. These expressions therefore exchange place in the formulae for the two rules when $N$ is odd.

\section{The error formula for an odd number of nodes}

We now turn to our aim, namely that of finding a formula for $C_{N}(f, h)-f$. One immediately sees that $C_{N}(f, h)$ may be written as [Val, Kre, Ber1]

$$
C_{N}(f, h)(x)=\frac{h}{\pi} \sin \frac{\pi}{h} x \sum_{n=-N}^{N}{ }^{\prime \prime}(-1)^{n} \frac{f_{n}}{x-x_{n}} .
$$

The right-hand expression may be interpreted as a difference of quadrature formulae of $\S 2$ with step $2 h$ :

$$
C_{N}(f, h)(x)=\frac{1}{2 \pi} \sin \left(\frac{\pi}{h} x\right)(-1)^{N}\left(T_{2 h}\left(I_{x}^{[-X, X]}\right)-M_{2 h}\left(I_{x}^{[-X, X]}\right)\right),
$$

(the factor $(-1)^{N}$ takes care of the sign at the extremal nodes in dependence on the parity of $N)$. But, with

$$
\widetilde{h}:=2 h
$$


and if $N$ is even, so that $T_{\widetilde{h}}$ and $M_{\widetilde{h}}$ cover an even number of intervals,

$$
\begin{aligned}
& T_{\widetilde{h}}\left(I_{x}^{[-X, X]}\right)= I_{x}^{[-X, X]}+\pi f(x) \cot \frac{\pi}{\widetilde{h}} x+\sum_{k=1}^{m+1} a_{2 k}(x) \widetilde{h}^{2 k} \\
&-\frac{\widetilde{h}^{2 m+2}}{(2 m+2) !} \int_{-X}^{X} F^{(2 m+2)}(y) \bar{P}_{2 m+2}\left(\frac{y}{\widetilde{h}}\right) d y \\
& M_{\widetilde{h}}\left(I_{x}^{[-X, X]}\right)=I_{x}^{[-X, X]}-\pi f(x) \tan \frac{\pi}{\widetilde{h}} x-\sum_{k=1}^{m+1}\left(1-2^{1-2 k}\right) a_{2 k}(x) \widetilde{h}^{2 k} \\
&-\frac{\widetilde{h}^{2 m+2}}{(2 m+2) !} \int_{-X}^{X} F_{2}^{(2 m+2)}(y) \bar{P}_{2 m+2}\left(\frac{1}{2}-\frac{y}{\widetilde{h}}\right) d y
\end{aligned}
$$

with $F_{2}$ as in (2.5). For odd $N$ the exchange of the derivative-free terms and of the values of $\bar{P}_{2 m+2}$ between $T_{\widetilde{h}}$ and $M_{\widetilde{h}}$ introduces another factor $-1=(-1)^{N}$ in their difference.

Subtracting $M_{\widetilde{h}}$ from $T_{\widetilde{h}}$, using the trigonometric identity $\tan \alpha+\cot \alpha=2 / \sin 2 \alpha$, and simplifying yield the first version of the error formula for $2 N+1$ nodes,

$$
\begin{aligned}
C_{N}(f, h)(x)-f(x)= & \frac{(-1)^{N}}{2 \pi} \sin \frac{2 \pi}{\widetilde{h}} x \sum_{k=1}^{m+1} b_{2 k}(x) \widetilde{h}^{2 k} \\
& \quad-\frac{\widetilde{h}^{2 m+2}}{(2 m+2) !} \int_{-X}^{X} F_{2}^{(2 m+2)}(y) \bar{Q}_{2 m+2}\left(\frac{y}{\widetilde{h}}\right) d y, \\
b_{2 k}(x):= & 2\left(1-4^{-k}\right) \frac{B_{2 k}}{(2 k) !}\left[\left(\frac{f(y)}{x-y}\right)^{(2 k-1)}(X)-\left(\frac{f(y)}{x-y}\right)^{(2 k-1)}(-X)\right]
\end{aligned}
$$

with $\bar{Q}_{k}(z):=(-1)^{N}\left(\bar{P}_{k}(z)-\bar{P}_{k}\left(\frac{1}{2}-z\right)\right)$.

The oscillatory sine-factor in front of the sum vanishes at every $x_{n}$, reflecting the interpolation property. On the other hand, it complicates the practical interpretation of the formula. For a given $x$, it is namely possible to pick a sequence of $h$ for which the factor is growing toward 1 from a value close to 0 . To avoid this, one may take a factor $\widetilde{h}$ in front of the sine to get

$$
C_{N}(f, h)(x)-f(x)=(-1)^{N} x \operatorname{sinc}\left(\frac{2 \pi}{\widetilde{h}} x\right)\left(\sum_{k=1}^{m+1} b_{2 k}(x) \widetilde{h}^{2 k-1}+\mathcal{O}\left(h^{2 m+1}\right)\right) .
$$

Now, since $\operatorname{sinc}\left(\frac{2 \pi}{\breve{h}} x\right) \rightarrow 0$ as $h \rightarrow 0$, the effect just mentioned asymptotically disappears: the polynomial part of the error decays with $h$ at least like a constant times sinc $\left(\frac{\pi}{h} x\right) h$. 
Summarizing we have the following.

\section{Theorem 3.1}

Let $f \in C^{2 m+1}[-X, X], X \in \mathbb{R}^{+}$, with $f^{(2 m+2)}$ absolutely integrable be interpolated on the interval $[-X, X]$ by the sinc interpolant $C_{N}(f, h)$ in (3.1) with $N \in \mathbb{N}, h=\frac{X}{N}, \widetilde{h}:=2 h$ and $x_{n}=n h$. Then the difference $C_{N}(f, h)-f$ is given by formulae (3.4) and (3.5) with $F_{2}$ from (2.5).

\section{Sinc interpolation between an even number of nodes}

In sinc interpolation, the case of an even number of points is certainly less common than that of an odd number. It might however have its importance, for instance when $f$ is periodic [Ber1] or with one-sided sinc interpolation [Ber4]. The finite cardinal series

$$
C_{N}^{(e)}(f, h)(x):=\sum_{n=-N+1}^{N} \prime \prime f\left(x_{n-\frac{1}{2}}\right) \operatorname{sinc}\left[\frac{\pi}{h}\left(x-x_{n-\frac{1}{2}}\right)\right], \quad x_{n-\frac{1}{2}}=\left(n-\frac{1}{2}\right) h,
$$

with $h=2 X /(2 N-1)$ then does not interpolate at 0 .

The main difference with the case of an odd number of points is the fact that the weights in the extreme terms of the sum now carry opposite signs. One may again write the sum as the difference of quadrature formulae for equidistant points, but these formulae are then asymmetric as one extremity is not a node.

We shall transform the interpolation problem into one with an odd number of nodes by first changing the variable and moving the points. For that purpose, consider again to every function $s$ on $[-X, X]$ its shift $\widehat{s}(x):=s(x-X)$, with domain $[0,2 X]$, and to every shifted $\widehat{s}$ its unshifted $s(x)=\widehat{s}(x+X)$. The sum in (4.1) is an expression in terms of the $\widehat{f}\left(x_{n}\right)$ : recalling $\widetilde{h}=2 h$, we have

$$
\begin{aligned}
C_{N}^{(e)}(f, h)(x) & =\frac{h}{\pi} \sum_{n=-N+1}^{N} \prime \prime f\left(x_{n-\frac{1}{2}}\right) \frac{\sin \left[\frac{\pi}{h} x-\left(n-\frac{1}{2}\right) \pi\right]}{x-x_{n-\frac{1}{2}}} \\
& =\frac{\widetilde{h}}{2 \pi} \cos \frac{\pi}{h} x \sum_{n=-N+1}^{N} \prime \prime f\left(x_{n-\frac{1}{2}}\right) \frac{(-1)^{n}}{x-x_{n-\frac{1}{2}}}
\end{aligned}
$$




$$
=\frac{\widetilde{h}}{2 \pi} \cos \frac{\pi}{h} x \sum_{n=0}^{2 N-1^{\prime \prime}} f\left(x_{n-\left(N-\frac{1}{2}\right)}\right) \frac{(-1)^{n-(N-1)}}{x-x_{n-\left(N-\frac{1}{2}\right)}}
$$

and, since $\left(N-\frac{1}{2}\right) h=X$,

$$
C_{N}^{(e)}(f, h)(x)=(-1)^{N-1} \frac{\tilde{h}}{2 \pi} \cos \frac{\pi}{h} x \sum_{n=0}^{2 N-1^{\prime \prime}} \widehat{f}\left(x_{n}\right) \frac{(-1)^{n}}{x+X-x_{n}} .
$$

To transform the problem into one with an odd number of points, we now extend $\widehat{f}(x)$ and $\frac{\widehat{f}(y)}{x-y}$ to $[-2 X, 0]$ as even functions, the latter by defining

$$
\widehat{g}_{x}(y):= \begin{cases}\frac{\widehat{f}(y)}{x-y}, & 0 \leq y \leq 2 X \\ -\frac{\widehat{f}(-y)}{-x-y}, & -2 X \leq y \leq 0\end{cases}
$$

which, by our shift convention, implies $g_{x}(y)=\widehat{g}_{x+X}(y+X)=f(y) /(x-y)$ on $[-X, X]$. Then

$$
\begin{aligned}
C_{N}^{(e)}(f, h)(x) & =\frac{(-1)^{N-1}}{2} \frac{\widetilde{h}}{2 \pi} \cos \frac{\pi}{h} x\left(\sum_{n=-(2 N-1)}^{0 "} \widehat{f}\left(-x_{n}\right) \frac{(-1)^{n}}{x+X+x_{n}}+\sum_{n=0}^{2 N-1^{\prime \prime}} \widehat{f}\left(x_{n}\right) \frac{(-1)^{n}}{x+X-x_{n}}\right), \\
& =\frac{(-1)^{N-1}}{4 \pi} \cos \frac{\pi}{h} x\left(\widetilde{h}_{n=-(2 N-1)}^{2 N-1} \sum^{\prime \prime}(-1)^{n} \widehat{g}_{x+X}\left(x_{n}\right)\right)
\end{aligned}
$$

(the double value at 0 eliminates the prime there) and with $\widehat{I}_{x}^{[-2 X, 2 X]}:=\mathrm{PV} \int_{-2 X}^{2 X} \widehat{g}_{x}(y) d y$

$$
=\frac{(-1)^{N-1}}{4 \pi} \cos \frac{\pi}{h} x\left(M_{\widetilde{h}}\left(\widehat{I}_{x+X}^{[-2 X, 2 X]}\right)-T_{\widetilde{h}}\left(\widehat{I}_{x+X}^{[-2 X, 2 X]}\right)\right),
$$

since the signs at the extremal nodes are now the same.

One may not continue as in (3.4), however, for now $\widehat{f}$, rolled up on a circle of diameter $\frac{4 X}{\pi}$ [Ber2], has two jumps instead of only one at $2 X \equiv-2 X$ : its derivatives usually are discontinuous at 0 . We thus need a generalization of Hunter's and Lyness' theorem for functions with several jumps, which will follow from modifying the proof in [Ber2] along the Lyness-Elliott lines [Ell].

First we introduce some notation. Let $f$ be piecewise $C^{q-1}[-X, X]$, i.e., $(q-1)$-times continuously differentiable on $[-X, X]$ except at interior jumps, at which the limits of $f$ and its $q-1$ derivatives exist on both sides. Denote by $c_{0}$ the point $-X \equiv X$ and by $c_{j}$, $j=1, \ldots, J$, the other jumps, and let $f$ be redefined at $c_{j}$ as the middle of the jump,

$$
f\left(c_{j}\right):=\frac{f\left(c_{j}-\right)+f\left(c_{j}+\right)}{2}, \quad j=0, \ldots, J,
$$


where, as usual, $f(x \pm):=\lim _{\epsilon \rightarrow 0} f(x \pm \epsilon)$, and $f\left(c_{0} \pm\right):=\lim _{\epsilon \rightarrow 0} f(\mp X \pm \epsilon)$.

We shall give the Euler-Maclaurin formula for equidistant Riemann sums

$$
R_{t}(h):=h \sum_{n=0}^{N-1} g(-X+(n+t) h)=h \sum_{n=1}^{N} g(-X+(n-1+t) h), \quad 0 \leq t<1, \quad h:=\frac{2 X}{N},
$$

of a Cauchy integral on the interval $[-X, X]$ (or, equivalently, on a circle of radius $\frac{X}{\pi}$ ), where the integrand may have several singularities to be integrated in the principal value sense, as $\widehat{g}_{x}$ at $x+X$ and $-(x+X)$ in (4.4). $t=0$ yields the trapezoidal rule, $t=1 / 2$ the midpoint rule. $t_{0}:=t$ obviously is the relative distance from the jump $-X \equiv X$ to the following integration node. Similarly, we determine for every other jump $c_{j}$ the interval that contains it, i.e., the index $n_{j}, 0 \leq n_{j} \leq N-1$, such that $c_{j} \in\left[-X+\left(n_{j}+t\right) h,-X+\left(n_{j}+1+t\right) h\right]$,

$j=0, \ldots, J$; this determines $t_{j}:=\frac{\left(n_{j}+1\right) h-c_{j}}{h}$, the relative location of $c_{j}$ with respect to the following node.

\section{Theorem 4.1 (Generalized Euler-Maclaurin formula for Cauchy integrals)}

Let $f$ be piecewise $C^{q-1}[-X, X], q \in \mathbb{N}, q \geq 2$, let $c_{j}, j=0, \ldots, J$, denote its jumps and $f\left(c_{j}\right)$ and $t_{j}$ be defined as above. Suppose that $f^{(q)}$ is integrable on the intervals between two jumps. Let $[-X, X)$ be partitioned into $L$ disjoint intervals $K_{\ell}=\left[c_{i}, c_{j}\right), \ell=1, \ldots, L$, such that $[-X, X)=\cup_{\ell=1}^{L} K_{\ell}$ and $g$ is given on $K_{\ell}$ by $g(y)=\frac{f(y)}{x_{\ell}-y}, K_{\ell} \ni \widehat{x}_{\ell} \neq-X+(n+t) h$, $n=0, \ldots, N-1$. Let $R_{t}(h)$ be any Riemann sum (4.5) of $P V \int_{-X}^{X} g(y) d y$.

Then the integration error may be written as

$$
\begin{array}{r}
R_{t}(h)-P V \int_{-X}^{X} g(y) d y=(-1)^{N} \pi \sum_{\ell=1}^{L} f\left(\widehat{x}_{\ell}\right) \operatorname{cta}\left[\pi\left(\frac{\widehat{x}_{\ell}}{h}-t\right)\right]+\sum_{k=1}^{q} a_{k} h^{k} \\
-\frac{h^{q}}{q !} \int_{-X}^{X} F_{2, L}^{(q)}(y) \sum_{j=0}^{J} \bar{P}_{q}\left(t_{j}-\frac{y+X}{h}\right) d y
\end{array}
$$

with

$$
\begin{aligned}
& \text { cta }:= \begin{cases}\cot , \quad N \text { even, } \\
\tan , \quad N \text { odd, }\end{cases} \\
& a_{1}:=\sum_{j=0}^{J} \theta_{j} P_{1}\left(t_{j}\right)\left[g\left(c_{j}-\right)-g\left(c_{j}+\right)\right], \quad \theta_{j}:= \begin{cases}0, & t_{j}=0, \\
1, & t_{j} \neq 0,\end{cases} \\
& a_{k}:=\sum_{j=0}^{J} \frac{P_{k}\left(t_{j}\right)}{k !}\left[g^{(k-1)}\left(c_{j}-\right)-g^{(k-1)}\left(c_{j}+\right)\right], \quad 2 \leq k \leq q,
\end{aligned}
$$




$$
F_{2, L}(y):=g(y)-\frac{\pi}{2 X} \sum_{\ell=1}^{L} f\left(\widehat{x}_{\ell}\right) \cot \left[\frac{\pi}{2 X}\left(\widehat{x}_{\ell}-y\right)\right]
$$

and where $\bar{P}_{k}$ again denotes the 1-periodic continuation of the Bernoulli polynomial of degree $k$.

A version of this theorem for the interval [0,1] and with $J=0$ is described in [Lyn]. When all jumps coincide with nodes $\left(t_{j}=0\right.$, all $\left.j>0\right)$, then for the trapezoidal rule $(t=0)$ all the coefficients $a_{k}$ with odd $k$ vanish: for $k=1, \theta_{j}=0$ for all $j$, and $P_{k}(0)=B_{k}=0$ for odd $k \geq 3$; (2.5) then is a special case of (4.6) with $N$ even, $J=0$ and $L=1$. With the midpoint rule $(t=1 / 2), P_{k}(1 / 2)=0$ for every odd $k$ and (4.6) again becomes the formula corresponding to (2.5) when $J=0$ and $L=1$.

When $q$ is even and $f \in C^{q}$, the last two terms of (4.6) might be combined into a single $\mathcal{O}\left(h^{q}\right)$-term.

We may now continue with (4.4). Here $T_{\widetilde{h}}$ and $M_{\widetilde{h}}$ cover the odd number $2 N-1$ of intervals. On the circle of diameter $\frac{4 X}{\pi}$, the extended $\widehat{f}$ has two jumps, $c_{0}=2 X \equiv-2 X$ and $c_{1}=0$. For $T_{\widetilde{h}}, c_{0}$ is at a node $\left(t_{0}=0, \theta_{0}=0\right)$ while $c_{1}$ lies in the center of an interval $\left(t_{1}=1 / 2, \theta_{1}=1\right) . a_{1}=0$ in view of $P_{1}(1 / 2)=0$ and the $a_{k}$ with odd $k \geq 3$ vanish too, for $P_{k}(0)=P_{k}(1 / 2)=0$. Thus in $(4.6)$

$$
\begin{aligned}
& T_{\widetilde{h}}\left(\widehat{I}_{x+X}^{[-2 X, 2 X]}\right)= R_{0}(\widetilde{h}) \\
&= \widehat{I}_{x+X}^{[-2 X, 2 X]}-\pi\left\{-\widehat{f}(-(x+X)) \tan \left[-\pi \frac{x+X}{\widetilde{h}}\right]+\widehat{f}(x+X) \tan \left[\pi \frac{x+X}{\widetilde{h}}\right]\right\} \\
& \quad+\sum_{k=1}^{m}\left\{\frac{P_{2 k}(0)}{(2 k) !}\left[\widehat{g}_{x+X}^{(2 k-1)}(2 X)-\widehat{g}_{x+X}^{(2 k-1)}(-2 X)\right]\right. \\
&\left.\quad+\frac{P_{2 k}(1 / 2)}{(2 k) !}\left[\widehat{g}_{x+X}^{(2 k-1)}(0-)-\widehat{g}_{x+X}^{(2 k-1)}(0+)\right]\right\} \widetilde{h}^{2 k}+\mathcal{O}\left(h^{2 m+2}\right)
\end{aligned}
$$

(the second negative sign in front of $\widehat{f}(x+X)$ comes from the definition (4.3) of $\left.\widehat{g}_{x}\right)$.

For $M_{\widetilde{h}}, c_{0}$ is between two nodes $\left(t_{0}=1 / 2, \theta_{0}=1\right)$, while $c_{1}$ is at a node $\left(t_{1}=0, \theta_{1}=0\right)$. Thus, again, $a_{k}=0$ for every odd $k$ and 


$$
\begin{aligned}
M_{\widetilde{h}}\left(\widehat{I}_{x+X}^{[-2 X, 2 X]}\right)= & R_{1 / 2}(\widetilde{h}) \\
= & \widehat{I}_{x+X}^{[-2 X, 2 X]}-\pi\left\{-\widehat{f}(-(x+X)) \tan \left[\pi\left(-\frac{x+X}{\widetilde{h}}-\frac{1}{2}\right)\right]\right. \\
+ & \left.\widehat{f}(x+X) \tan \left[\pi\left(\frac{x+X}{\widetilde{h}}-\frac{1}{2}\right)\right]\right\} \\
+ & \sum_{k=1}^{m}\left\{\frac{P_{2 k}(1 / 2)}{(2 k) !}\left[\widehat{g}_{x+X}^{(2 k-1)}(2 X)-\widehat{g}_{x+X}^{(2 k-1)}(-2 X)\right]\right. \\
& \left.\quad+\frac{P_{2 k}(0)}{(2 k) !}\left[\widehat{g}_{x+X}^{(2 k-1)}(0-)-\widehat{g}_{x+X}^{(2 k-1)}(0+)\right]\right\} \widetilde{h}^{2 k}+\mathcal{O}\left(h^{2 m+2}\right) .
\end{aligned}
$$

But with $\widehat{f}$ and $\widehat{g}$ even (thus their odd order derivatives odd),

$$
\begin{aligned}
& T_{\widetilde{h}}\left(\widehat{I}_{x+X}^{-2 X, 2 X]}\right)= \widehat{I}_{x+X}^{[-2 X, 2 X]}-2 \pi \tan \left[\pi\left(\frac{x+X}{\widetilde{h}}\right)\right] \widehat{f}(x+X) \\
&+2 \sum_{k=1}^{m}\left[\frac{P_{2 k}(0)}{(2 k) !} \widehat{g}_{x+X}^{(2 k-1)}(2 X)-\frac{P_{2 k}(1 / 2)}{(2 k) !} \widehat{g}_{x+X}^{(2 k-1)}(0+)\right] \widetilde{h}^{2 k}+\mathcal{O}\left(h^{2 m+2}\right) \\
& M_{\widetilde{h}}\left(\widehat{I}_{x+X}^{[-2 X, 2 X]}\right)= \widehat{I}_{x+X}^{[-2 X, 2 X]}+2 \pi \cot \left[\pi\left(\frac{x+X}{\widetilde{h}}\right)\right] \widehat{f}(x+X) \\
&+2 \sum_{k=1}^{m}\left[\frac{P_{2 k}(1 / 2)}{(2 k) !} \widehat{g}_{x+X}^{(2 k-1)}(2 X)-\frac{P_{2 k}(0)}{(2 k) !} \widehat{g}_{x+X}^{(2 k-1)}(0+)\right] \widetilde{h}^{2 k}+\mathcal{O}\left(h^{2 m+2}\right)
\end{aligned}
$$

and (4.4) becomes

$$
\begin{aligned}
C_{N}^{(e)}(f, h)(x)= & \frac{(-1)^{N-1}}{4 \pi} \cos \frac{\pi}{h} x\left\{4 \pi \widehat{f}(x+X) / \sin \left[2 \pi\left(\frac{x+X}{\widetilde{h}}\right)\right]\right. \\
& \left.+2 \sum_{k=1}^{m} \frac{P_{2 k}(1 / 2)-B_{2 k}}{(2 k !)}\left[\widehat{g}_{x+X}^{(2 k-1)}(0+)+\widehat{g}_{x+X}^{(2 k-1)}(2 X)\right] \widetilde{h}^{2 k}\right\}+\mathcal{O}\left(h^{2 m+2}\right) .
\end{aligned}
$$

Now $\left.\sin \left[2 \pi\left(\frac{x+X}{\widetilde{h}}\right)\right]=\sin \left[\pi\left(\frac{x+X}{h}\right)\right]=\sin \left[\frac{\pi}{h} x+(N-1 / 2) \pi\right)\right]=-(-1)^{N} \cos \frac{\pi}{h} x, \widehat{f}(x+$ $X)=f(x), \widehat{g}_{x+X}^{(2 k-1)}(0+)=g_{x}^{(2 k-1)}(-X), \widehat{g}_{x+X}^{(2 k-1)}(2 X)=g_{x}^{(2 k-1)}(X)$ and we have the following formula.

\section{Theorem 4.2}

Let $f \in C^{2 m+1}[-X, X], X \in \mathbb{R}^{+}$, with $f^{(2 m+2)}$ absolutely integrable be interpolated on the interval $[-X, X]$ by the sinc interpolant $C_{N}^{(e)}(f, h)$ in (4.1) with $N \in \mathbb{N}, h=\frac{2 X}{2 N-1}$ and $x_{n-\frac{1}{2}}=\left(n-\frac{1}{2}\right) h$. Then the difference $C_{N}^{(e)}(f, h)-f$ is given by the formula 


$$
\begin{gathered}
C_{N}^{(e)}(f, h)(x)-f(x)=\frac{(-1)^{N}}{2 \pi} \cos \frac{2 \pi}{\widetilde{h}} x \sum_{k=1}^{m} \frac{B_{2 k}-P_{2 k}(1 / 2)}{(2 k) !}\left[g_{x}^{(2 k-1)}(-X)+g_{x}^{(2 k-1)}(X)\right] \widetilde{h}^{2 k} \\
+\mathcal{O}\left(h^{2 m+2}\right),
\end{gathered}
$$

with $g_{x}(y)=f(y) /(x-y)$ and $\widetilde{h}=2 h$.

The factors $\frac{B_{2 k}-P_{2 k}(1 / 2)}{(2 k) !}$ are the same as in formula (3.4) for the case of an odd number of points. The sole changes from that formula (besides in the rest term) are the replacement of the sine by the cosine, and of the differences of end-point derivatives by their sums.

One may again take a factor $\widetilde{h}$ in front of the sum to annihilate a possible increase of the cosine for particular (decreasing) sequences of $h$.

In [Ber4] we give first applications of formulae (3.4) and (4.7). Here we just notice that they cannot be used directly for evaluating and/or correcting the error at $x$ too close to the endpoints $-X$ and $X$ of the interval: the derivatives of $\frac{f(y)}{x-y}$ become much larger than $1 / \tilde{h}$ there.

\section{Appendix: an alternate proof of formula (4.7)}

The proof of (4.7) given above makes a detour through a problem involving an odd number of points and formula (3.4) and requires a change of variable. We shall now give a direct proof that brings Riemann sums other than $T$ and $M$ into play.

Let us go back to formula (4.2). Roll the interval $[-X, X]$ up on the right half circle of diameter $\frac{4 X}{\pi}$ about 0 [Ber2] and consider the functions $f(y)$ and $g(y):=\frac{f(y)}{x-y}$ on that half-circle. Then extend $f$ and $g$ to the left-hand half-circle as even functions with respect to $X$ (and thus automatically with respect to $-X$ ). By associating the weights 1 and -1 with the points $x_{n}$ on the circle (see the figure) and using $\int_{-2 X}^{-X}+\int_{-X}^{X}+\int_{X}^{2 X}=\int_{-2 X}^{2 X}$, one sees that in $(4.2)$

$$
\widetilde{h} \sum_{n=-N+1}^{N} \prime \prime f\left(x_{n-\frac{1}{2}}\right) \frac{(-1)^{n}}{x-x_{n-\frac{1}{2}}}=\frac{1}{2}\left[R_{1 / 4}(\widetilde{h})-R_{3 / 4}(\widetilde{h})\right],
$$

where $R_{t}(h)$ is the Riemann sum given similarly to formula (4.5) by

$$
R_{t}(h):=h \sum_{n=0}^{2 N-1} g(-2 X+(n+t) h),
$$


$t$ being again the relative distance from the left extremity of the interval to the first node. (The factor $1 / 2$ takes care of the fact that $R_{t}$ approaches the integral $I_{x}^{[-2 X, 2 X]}=2 I_{x}^{[-X, X]}$.)

The function $f$ now has two jumps $c_{1}=-X$ and $c_{2}=X$ (notice that it does not have any at 0 nor at the extremities $-2 X \equiv 2 X$ ). If $N$ is even, then $-X$ is not a node of $R_{1 / 4}$, for which it lies at the midpoint of two nodes, but is one for $R_{3 / 4}$; it is the other way around for $N$ odd.

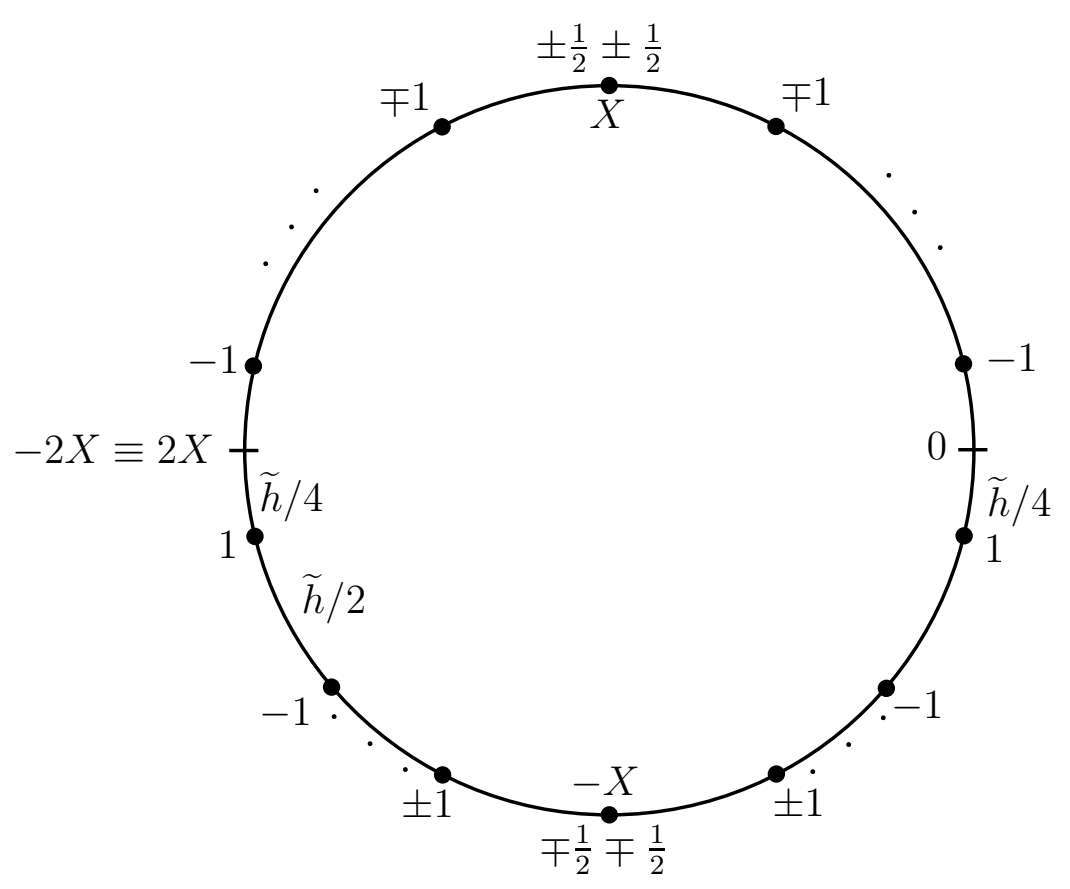

Nodes and weights for the quadrature rules in $\S 5$.

When two signs appear, the top one is for $N$ even, the other for $N$ odd.

We now apply Theorem 4.1 to $R_{1 / 4}$ and $R_{3 / 4}$. Since $g$ is even, all differences of even order derivatives, thus all coefficients of odd powers of $h$, vanish. If $N$ is even, then, for $R_{1 / 4}$, $t_{1}=\frac{1}{2}$ and $t_{2}=0$; it is the opposite for $R_{3 / 4}$. For $x>0$ the singularities to be integrated in the principal value sense are at $x$ and $2 X-x$, for $x<0$ at $x$ and $-2 X-x \equiv 2 X-x \bmod 4 X$, thus at the same locations. Thus

$$
\begin{aligned}
R_{1 / 4}=I_{x}^{[-2 X, 2 X]}+ & \pi\left\{f(x) \cot \left[\pi\left(\frac{x}{\widetilde{h}}-\frac{1}{4}\right)\right]-f(2 X-x) \cot \left[\pi\left(\frac{2 X-x}{\widetilde{h}}-\frac{1}{4}\right)\right]\right\} \\
+ & \sum_{k=1}^{m}\left\{\frac{P_{2 k}(0)}{(2 k) !}\left[g^{(2 k-1)}(-X-)-g^{(2 k-1)}(-X+)\right]\right.
\end{aligned}
$$




$$
\left.+\frac{P_{2 k}(1 / 2)}{(2 k) !}\left[g^{(2 k-1)}(X-)-g^{(2 k-1)}(X+)\right]\right\} \widetilde{h}^{2 k}+\mathcal{O}\left(h^{2 m+2}\right)
$$

(recall that $-f$ appears in the numerator of the even $g$ on the left half-circle). But $2 X=$ $(2 N-1) h$, thus $\frac{2 X}{\widetilde{h}}=N-1 / 2$ and $\cot \left[\pi\left(\frac{2 X-x}{\widetilde{h}}-\frac{1}{4}\right)\right]=\cot \left[-\frac{\pi}{\breve{h}} x-\frac{3 \pi}{4}\right]=\cot \left[-\frac{\pi}{\widetilde{h}} x+\frac{\pi}{4}\right] ;$ moreover, in view of the parity of $f$ and $g, f(2 X-x)=f(x)$ and $g^{(2 k-1)}( \pm X-)=$ $-g^{(2 k-1)}( \pm X+)$; thus

$$
\begin{aligned}
R_{1 / 4}=I_{x}^{[-2 X, 2 X]}+2 \pi f(x) \cot \left[\pi\left(\frac{x}{\widetilde{h}}-\frac{1}{4}\right)\right] \\
\quad+\sum_{k=1}^{m} \frac{2}{(2 k) !}\left[B_{2 k} g^{(2 k-1)}(X)-P_{2 k}(1 / 2) g^{(2 k-1)}(-X)\right] \widetilde{h}^{2 k}+\mathcal{O}\left(h^{2 m+2}\right) .
\end{aligned}
$$

Similarly

$$
\begin{aligned}
& R_{3 / 4}=I_{x}^{[-2 X, 2 X]}+ \pi f(x)\left\{\cot \left[\pi\left(\frac{x}{\widetilde{h}}-\frac{3}{4}\right)\right]-\cot \left[\pi\left(N-\frac{1}{2}-\frac{x}{\widetilde{h}}-\frac{3}{4}\right)\right]\right\} \\
&+\sum_{k=1}^{m}\left\{\frac{P_{2 k}(0)}{(2 k) !}\left[g^{(2 k-1)}(-X-)-g^{(2 k-1)}(-X+)\right]\right. \\
&\left.\quad+\frac{P_{2 k}(1 / 2)}{(2 k) !}\left[g^{(2 k-1)}(X-)-g^{(2 k-1)}(X+)\right]\right\} \widetilde{h}^{2 k}+\mathcal{O}\left(h^{2 m+2}\right) \\
&=2 \pi f(x) \cot \left[\pi\left(\frac{x}{\widetilde{h}}+\frac{1}{4}\right)\right] \\
& \quad+\sum_{k=1}^{m} \frac{2}{(2 k) !}\left[P_{2 k}(1 / 2) g^{(2 k-1)}(X)-B_{2 k} g^{(2 k-1)}(-X)\right] \widetilde{h}^{2 k}+\mathcal{O}\left(h^{2 m+2}\right),
\end{aligned}
$$

so that with $(5.1)$ in $(4.2)$

$$
\begin{aligned}
C_{N}^{(e)}(f, h)(x)= & \frac{1}{2 \pi} \cos \frac{\pi}{h} x \cdot \frac{1}{2}\left[R_{1 / 4}(\widetilde{h})-R_{3 / 4}(\widetilde{h})\right] \\
= & \frac{1}{2 \pi} \cos \frac{\pi}{h} x\left\{\pi f(x)\left\{\cot \left[\pi\left(\frac{x}{\widetilde{h}}+\frac{1}{4}\right)\right]-\cot \left[\pi\left(\frac{x}{\widetilde{h}}-\frac{1}{4}\right)\right]\right\}\right. \\
& \left.\quad+\sum_{k=1}^{m} \frac{B_{2 k}-P_{2 k}(1 / 2)}{(2 k) !}\left[g^{(2 k-1)}(-X)+g^{(2 k-1)}(X)\right] \widetilde{h}^{2 k}\right\}+\mathcal{O}\left(h^{2 m+2}\right) .
\end{aligned}
$$

But $\cot \left[\pi\left(\frac{x}{h}+\frac{1}{4}\right)\right]-\cot \left[\pi\left(\frac{x}{h}-\frac{1}{4}\right)\right]=2 / \cos \frac{\pi}{h} x$; moreover, when $N$ is odd the $t_{j}$ 's are exchanged in all formulae, which permutes $P_{2 k}(0)$ and $P_{2 k}(1 / 2)$ and introduces the factor $(-1)^{N}$ to yield formula (4.7). 
Acknowledgment. The author thanks the referees for their numerous improvements in the form of this work.

\section{References}

[Ber1] Berrut J.-P., Barycentric formulae for cardinal (SINC-)Interpolants, Numer. Math. 54 (1989) 703-718. (Erratum 55 (1989) 747.)

[Ber2] Berrut J.-P., A circular interpretation of the Euler-Maclaurin formula, J. Comput. Appl. Math. 189 (2006) 375-386.

[Ber3] Berrut J.-P., A formula for the error of finite sinc-interpolation over a finite interval, Numer. Algorithms 45 (2007) 369-374.

[Ber4] Berrut J.-P., First applications of a formula for the error of finite sinc interpolation, Numer. Math. 112 (2009) 341-361.

[Bri-Hen] Briggs W. L., Henson V. E., The DFT: An Owner's Manual for the Discrete Fourier Transform (SIAM, Philadelphia, 1995).

[But-Spl-Ste] Butzer P. L., Splettstösser W., Stens R. L., The sampling theorem and linear prediction in signal analysis, Jber. d. Dt. Math.-Verein. 90 (1988) 1-70.

[But-Ste] Butzer P. L., Stens R. L., Sampling theory for not necessarily band-limited functions: a historical overview, SIAM Rev. 34 (1992) 40-53.

[Dav-Rab] Davis P. J., Rabinowitz P., Methods of Numerical Integration (2 ${ }^{\text {nd }}$ ed., Academic Press, San Diego, 1984).

[Ell] Elliott D., The Euler-Maclaurin formula revisited, J. Austral. Math. Soc. B 40 (Electronic) (1998) E27-E76.

[Hig1] Higgins J. R., Five short stories about the cardinal series, Bull. A.M.S. 12 (1985) 45-89.

[Hig2] Higgins J. R., Sampling Theory in Fourier and Signal Analysis: Foundations (Clarendon Press, Oxford, 1996).

[Hun] Hunter D. B., The numerical evaluation of Cauchy principal values of integrals by Romberg integration, Numer. Math. 21 (1973) 185-192.

[Kin-Che] Kincaid D., Cheney W., Numerical Analysis, Mathematics of Scientific Computing (Wadsworth, Belmont, 1991). 
[Kre] Kress R., Interpolation auf einem unendlichen Intervall, Computing 6 (1970) 274-288.

[Lun-Bow] Lund J., Bowers K. L., Sinc Methods for Quadrature and Differential Equations (SIAM, Philadelphia, 1992).

[Lyn] Lyness J. N., The Euler Maclaurin expansion for the Cauchy principal value integral, Numer. Math. 46 (1985) 611-622.

[Par] Partington J. R., Interpolation, Identification and Sampling (Clarendon Press, Oxford, 1997).

[Sch] Schwarz H. R., Numerische Mathematik (4 ${ }^{\text {te }}$ Aufl., Teubner, 1997; english translation of the second edition: Numerical Analysis. A Comprehensive Introduction, Wiley, New York, 1989).

[Ste] Stenger F., Numerical Methods based on Sinc and Analytic Functions (Springer, New York, 1993).

[Val] de la Vallée Poussin C. J., Sur la convergence des formules d'interpolation entre ordonnées équidistantes, Bull. de la Classe des Sci. de l'Acad. Roy. de Belgique Série 4 (1908) 319-410. 